\title{
WFDC2 GENE POLYMORPHISM IN PATIENTS WITH OVARIAN MASSES
}

\author{
Egunova M.A. ${ }^{1}$, Bragina E.Y. ${ }^{2}$, Kutsenko I.G. ${ }^{1}$ \\ ${ }^{1}$ Siberian State Medical University, Health Ministry of Russian Federation,Tomsk, e-mail: mariyaegunova@mail.ru; \\ ${ }^{2}$ Research Institute of Medical Genetics, Tomsk National Research Medical Center of the Russian Academy of Sciences, \\ Tomsk, e-mail: elena.bragina72@gmail.com
}

Aims.

To identify the relationship between polymorphic variants (rs2239533, rs2072956) of the WFDC2 gene and the presence of benign and malignant ovarian masses.

Materials and methods.

A comparative molecular genetic study of polymorphic variants (rs2239533, rs2072956) of the WFDC2 gene in patients with the presence of benign and malignant ovarian masses was carried out. The study included 55 patients with ovarian masses and 40 healthy women. Benign ovarian masses were histologically verified in 38 (69.1\%) patients of the main group, and malignant - in 17 (30.9\%). Tumour marker HE-4 was characterised by high specificity $(\mathbf{9 7 . 4 \%})$ at a relatively low sensitivity $(\mathbf{5 2 . 9 \%})$.

Results.

The molecular genetic study revealed a statistically significant predominance of the T/T genotype of polymorphic variant rs2239533 of the WFDC2 gene in patients with ovarian masses compared to the group of healthy women. After subdivision of the main study group into 2 subgroups based on the histological variants of tumours (benign or malignant), it was determined that carriage of the T/T genotype of variant rs2239533 of the WFDC2 gene was associated with the presence of malignant forms $(\mathrm{OR}=5.54(95 \% \mathrm{CI} 1.12-27.54), \mathrm{p}=0.03)$.

Conclusions.

Carrying the T/T genotype of variant rs2239533 of the WFDC2 gene is associated with the presence of malignant ovarian masses.

Keywords: ovarian mass, differential diagnosis, tumour marker, HE-4, polymorphism,

WFDC2, rs2239533, rs2072956

\section{INTRODUCTION.}

Today, outpatient differential diagnosis of benign and malignant ovarian tumors (BOTs and MOTs) uses the standard CA-125 tumor markers complemented with testing the blood serum for the concentration of HE-4 oncological markers. Previously published data suggests that an increase in CA-125 can be detected earlier in MOT patients than a statistically significant increase in HE-4 levels [1,2], which means that HE-4 is a more sensitive marker of early ovarian cancer (OC) stages than CA-125 (82.7\% vs. 45.9\%, respectively) [2]. Besides, some studies have shown that in nearly $50 \%$ of OC cases, HE-4 exceeded reference levels while CA-125 was within the discriminatory zone (DZ) $[1,2]$.

HE-4 is a product of the WFDC2 gene (WAP four-disulfide core domain protein 2) and is an acidic protein suggested to inhibit trypsin [3]. WFDC2 studies are isolated and dissimilar. A 2014 paper [4] showed that excess WFDC2 expression in the epithelial MOT cells stimulated tumor growth and progression. Another study detected higher WFDC2 expression in high-grade ovarian carcinomas than in their low-grade counterparts [5]. Thus, researchers identified a connection between higher WFDC2 expression and a more aggressive clinical course of the disease [4, 5]. Integrating whole-genome microarray data showed considerable WFDC2 expression in patients with serous epithelial ovarian cancer [6]. Comparing the WFDC2 expression in normal ovarian tissue, unaltered fallopian tubes, and samples of epithelial ovarian tumors extracted during primary 
(pre-chemotherapy) surgeries showed significantly higher expression in epithelial MOTs compared to unaffected ovaries ( $\mathrm{p}=0.000016)$. WFDC2 transcription activity was comparable in epithelial OC and in normal fallopian tubes $(\mathrm{p}=1.00)$. No correlation has been found between increased HE-4 levels and high WFDC2 expression despite the fact that both indicators independently correlate with adverse prognosis and low survival rates; this is an unexpected discovery that demands further research and explanation [7]. Neither Russian nor international literature has data on how WFDC2 polymorphisms may affect the tumor marker levels in the serum.

The goal hereof was to find how WFDC2 polymorphisms (rs2239533 and rs2072956) could correlate with benign and malignant ovarian tumors.

MATERIALS AND METHODS. Ninety-five Caucasian women (55 with ovarian tumors (OT) and 40 controls) underwent molecular genetic testing. The study involved 55 patients hospitalized to the gynecology clinic, Siberian State Medical University of the Ministry of Health of Russia, or to the Tomsk Regional Oncology Center, for ovarian tumor surgery; the 40 control group patients did not have pathologies according to the gynecologist's examination and pelvic ultrasound.

The study protocol was approved by the Ethics Committee of the Siberian State Medical University, Ministry of Health of Russia (Minutes No. 4336 of November 30, 2015); each enrolled patient signed informed consent.

All the patients underwent:

1) examination pursuant to the ovarian tumor standard (Order of the Ministry of Health of the Russian Federation No. 572n dd. November 1, 2012 On the Approval of Medical Care Procedures in Obstetrics and Gynecology Except the Use of Assisted Reproduction Technologies);

2) testing for HE-4 levels by chemiluminescent immunoassay (ARCHITECT, Abbott);

3) whole-blood sampling to extract DNA by phenol-chloroform extraction per the standard procedure [8] and to detect rs2239533 and rs2072956 WFDC2 polymorphisms;

4) histological testing of the surgical samples (the treatment group only);

5) data was processed statistically by means of SPSS Version 20.

Moscow-based DNK-Sintez provided primer design and synthesis for the molecular genetic testing. A real-time CFX Connect (Bio-Rad) automatic thermocycler was used for real-time PCR amplification in full compliance with the primer manufacturer's manuals. The amplification procedure comprised pre-denaturation at $95{ }^{\circ} \mathrm{C}$ over 3 minutes followed by 40 cycles of ignition (95 ${ }^{\circ} \mathrm{C}, 20$ seconds), chain elongation $\left(55^{\circ} \mathrm{C}, 30\right.$ seconds), and denaturation $\left(72{ }^{\circ} \mathrm{C}, 40\right.$ seconds).

SPSS tables were made for statistical processing. The team ran the Shapiro-Wilk test for distribution normality. Mean \pm standard deviation $(\mathrm{M} \pm \sigma)$ values were used to describe normally distributed quantitative data. Median and quartile (Me [Q25; Q75]) were used otherwise. Yatescorrected chi-square testing was applied to find whether the observed frequencies of the 
polymorphism-based genotypes matched their expected frequencies in compliance with the HardyWeinberg model. Chi-square or Fisher's exact test (if at least one cell of the contingency tables had $<5$ observations) were used to compare the allele and genotype occurrence rates in the studied groups. The nonparametric Mann-Whitney test identified intergroup differences. The odds ratio (OR) was computed at $95 \%$ CI to associate different genotypes with BOT or MOT. At OR $<1$, the association between the features was deemed negative; at $\mathrm{OR}>1$, positive. For statistical hypothesis testing, $\mathrm{p}=0.05$. Tumor marker sensitivity (SE) was calculated by the formula: $\mathrm{SE}=\mathrm{TP} / \mathrm{D}^{-} \times 100 \%$, where TP are true positive test results, $\mathrm{D}^{-}$is the number of patients. Method specificity (SP) was calculated by the formula: $\mathrm{SP}=\mathrm{TN} / \mathrm{D} \times 100 \%$, where $\mathrm{TN}$ is the number of true negative test results, $\mathrm{D}$ is the number of unaffected persons.

RESULTS. The treatment group comprised 55 patients with ovarian tumors aged 19 to 79 (48.5 \pm 2.2$)$. Twenty-seven (49\%) were of reproductive age, and 28 (51\%) were postmenopausal women. Histological testing of surgical samples identified BOT in 38 patients $(69.1 \%$ ) and MOT in $17(30.9 \%)$. Epithelial ovarian tumors prevailed in the treatment group at $74.6 \%$; there were also sex cord-gonadal stromal tumors (3.6\%), germ-cell tumors $(9.1 \%)$, and tumor-like lesions and processes in the ovaries $(12.7 \%)$.

HE-4 varied from 12.6 to $1,500(47.2[36.0 ; 87.7]) \mathrm{pmol} / \mathrm{l}$ in the treatment group. Patients of reproductive age had HE-4 concentrations of 12.6 to 922 (40.4 [32.7; 68.0]) pmol/l (DZ=70 pmol/l); postmenopausal OT women had 37.3 to $1,500(56.6[43 ; 308.3]) \mathrm{pmol} / \mathrm{l}(\mathrm{DZ}=140 \mathrm{pmol} / \mathrm{l})$. Ten patients of the treatment group had HE-4 levels above the upper reference limit; OC was confirmed by postoperative histological testing in 9 of them, while 1 had a follicular ovarian cyst combined with a corpus luteum cyst. Thus, HE-4 sensitivity was $52.9 \%$, specificity was $97.4 \%$.

HE false negatives were reported for $8(47.1 \%)$ MOT patients that had postmenopausal serous carcinoma $(n=4)$, mucinous carcinoma at reproductive age $(n=3)$, and postmenopausal clearcell carcinoma $(n=1)$. Earlier studies had shown that HE-4 was indeed low in the serum of patients that had endometrioid and clear-cell ovarian adenocarcinomas or mucinous OC, which was due to the source of such tumors [9-11].

The control group comprised 40 women aged 21 to 78 (50.5 [24.3;60.0]), of whom 20 $(50 \%)$ were of reproductive age and $20(50 \%)$ were menopausal. In this group, HE-4 varied from 22.4 to 84.9 (40.2 [35.9; 52.3]) pmol/1. Reproductive-age patients had HE-4 levels of 22.4 to 50.2 (36 [31.6; 41.0]) pmol/l, while their postmenopausal counterparts had 37.7 to 84.9 (51.1 [40.1; 57.8]) pmol/l. No false positives were reported for the controls.

For genetic testing, the research team picked two WFDC2 polymorphisms (rs2239533 and rs2072956) that were tested in two samples different in terms of OT presence.

In both groups, the genotypes based on the studied polymorphisms occurred in line with the Hardy-Weinberg model ( $\mathrm{p}>0.05)$.

Comparative analysis of rs2239533 prevalence identified statistically significantly $\left(\chi^{2=} 4.671\right.$, 
$\mathrm{p}=0.031)$ more frequent occurrence of the homozygous $\mathrm{T} / \mathrm{T}$ genotype $(78.2 \%)$ and less frequent occurrence of the heterozygous $\mathrm{T} / \mathrm{C}$ genotype (20.0\%) in the treatment group compared to the controls $(57.5 \%, 42.5 \%$, respectively), see Figure 1. Calculating the odds ratio OR showed that the $\mathrm{T} / \mathrm{T}$ genotype was indeed predisposed to OT (OR-2.649, (95\% CI, 1.081-6.487), $\mathrm{p}=0.042$ ). Genotypes were not found to be linked to OT histotypes ( $p>0.05$ ).

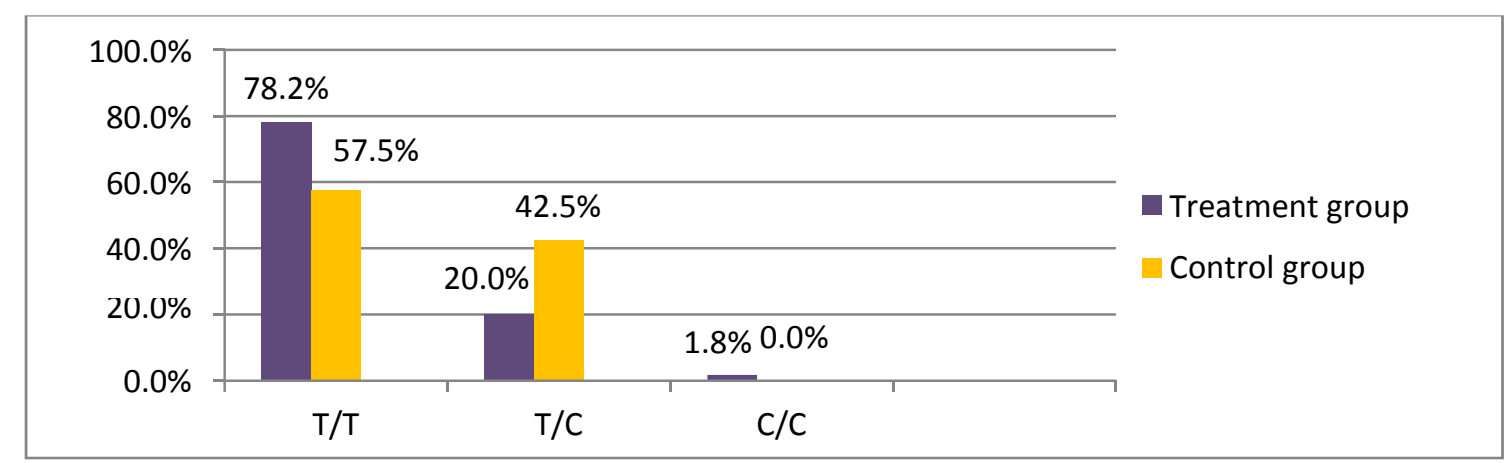

Figure 1. WFDC2 rs2239533 polymorphism occurrence in treated patients and controls

rs2072956 genotypes were distributed as follows in the treatment group: 40 patients with the $\mathrm{C} / \mathrm{C}$ genotype (72.7\%), 14 with $\mathrm{C} / \mathrm{G}(25.5 \%)$ and 1 with $\mathrm{G} / \mathrm{G}(1.8 \%) . \mathrm{C} / \mathrm{C}$ was nearly as frequent in the control group $(72.5 \%, \mathrm{n}=29), \mathrm{C} / \mathrm{G}$ was found in $11(27.5 \%)$ patients, see Figure 2. No

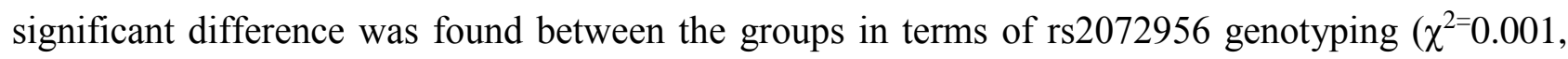
$\mathrm{p}=0.981)$, genotypes and OT occurrence did not correlate (OR=1, 011 (95\% CI 0.406-2.521), $\mathrm{p}=0.466)$.

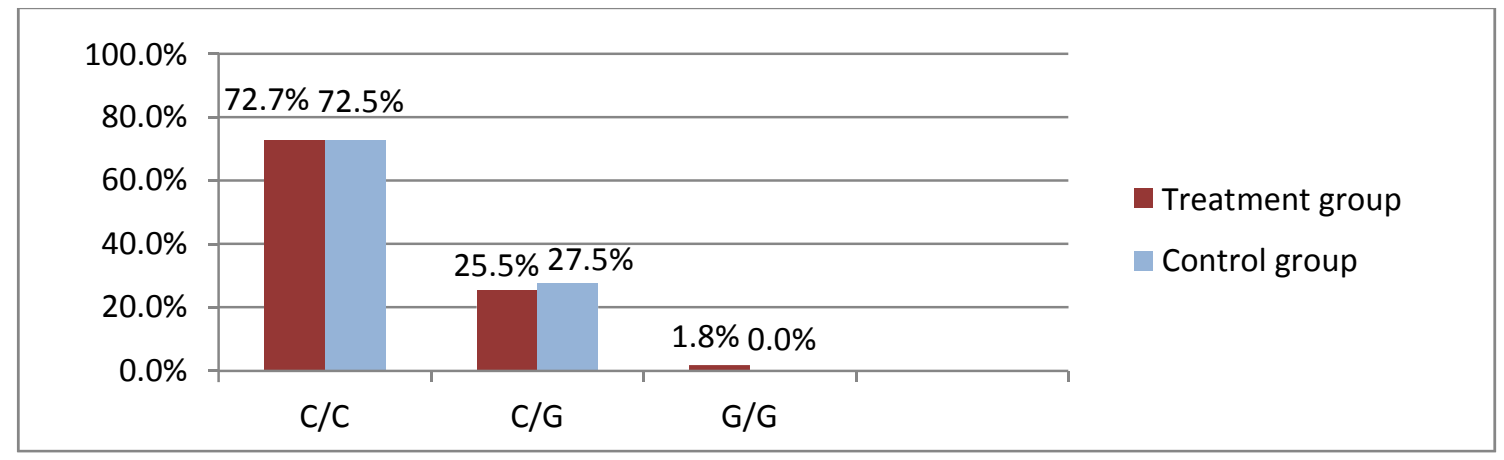

Figure 2. WFDC2 rs2072956 polymorphism occurrence in treated patients and controls

Next, the treatment group was split into two subgroups (1 with BOTs and 1 with MOTs).

Figures 3 and 4 show the genotypic distribution of rs2239533 and rs2072956 polymorphisms in BOT and MOT patients, respectively. 


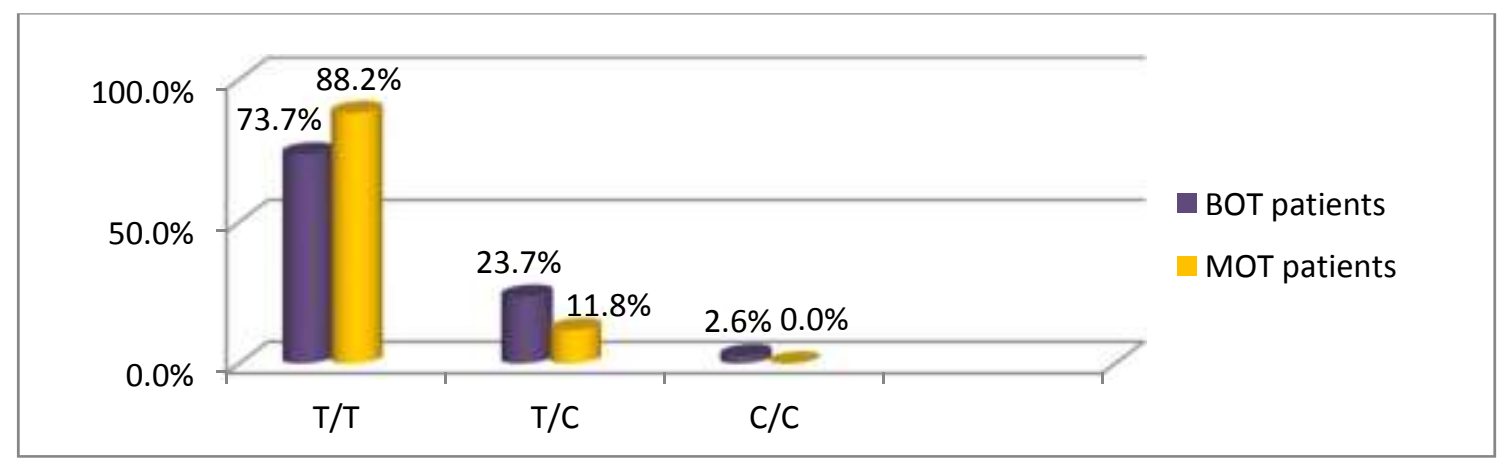

Figure 3. WFDC2 rs2239533 polymorphism occurrence in BOT and MOT patients

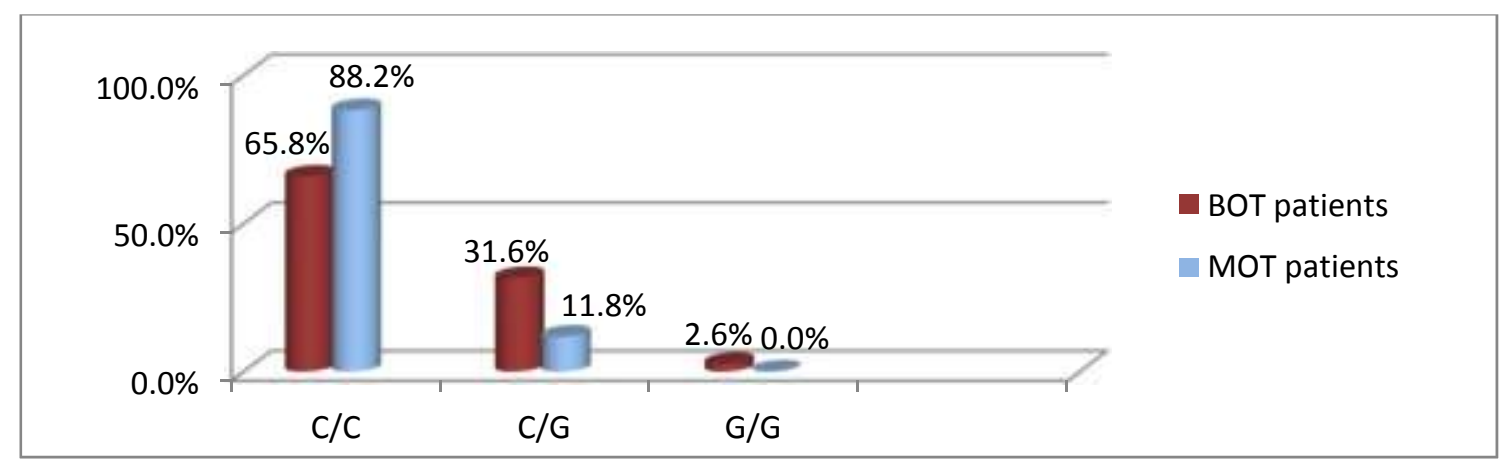

Figure 4. WFDC2 rs2072956 polymorphism occurrence in BOT and MOT patients

Genotype distribution did not differ significantly between BOT and MOT patients (for

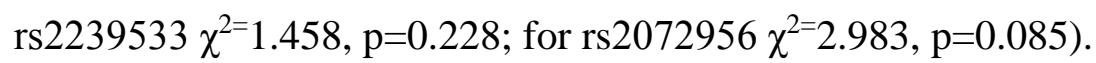

Comparing the occurrence rates of WFDC2 rs2239533 genotypes between the BOT/MOT subgroups and the controls showed that MOT patients were more prone to have T/T (88.2\%) and

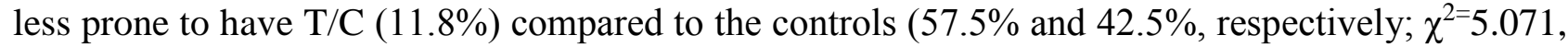
$\mathrm{p}=0.025) . \mathrm{rs} 2239533 \mathrm{~T} / \mathrm{T}$ genotype carriers are therefore at higher MOT risk $(\mathrm{OR}=5.54(95 \% \mathrm{CI}$ 1.12-27.54), $\mathrm{p}=0.03)$.

The polymorphic genotypes did not correlate with the HE-4 marker levels in the serum ( $p=0.153$ for $r s 2239533 ; p=0.549$ for $r s 2072956)$. However, it was noted that all the patients that had HE-4 levels above the DZ also had the rs2239533 T/T genotype and the rs2072956 C/C genotype.

The sample of this study is extremely small; however, its results could motivate further research into WFDC2 polymorphisms.

CONCLUSIONS. Molecular genetic testing showed that OT patients had significantly higher rs22239533 T/T genotype occurrence than the controls $\left(\chi^{2}=4.671, \mathrm{p}=0.031\right)$. Patients were split into histology-based subgroups (BOT and MOT groups) to further find that rs2239533 T/T genotype carriers were prone to have MOTs $(\mathrm{OR}=5.54$ (95\% $\mathrm{Ci} 1.12-27.54), \mathrm{p}=0.03)$. This requires further research and refinement on a larger sample. 
FINANCIAL SUPPORT AND SPONSORSHIP

Nil.

\section{CONFLICTS OF INTEREST}

The authors declare no conflict of interest

\section{SUPPLEMENTARY DATA (DOI)}

\section{REFERENCES}

1. Bast R.C., Skates S., Lokshin A. et al. Differential diagnosis of pelvic mass: improved algorithms and novel biomarkers. International Journal of Gynecological Cancer, 2012, vol. 22, pp. 5-8, doi 10.1097/igc.0b013e318251c97d.

2. Havrilesky L.J., Whitehead C.M., Rubatt J.M. et al. Evaluation of biomarker panels for early stage ovarian cancer detection and monitoring for disease recurrence. Gynecologic Oncology. 2008. Vol. 110, N 3, pp. 374-382, 10.1016/j.ygyno.2008.04.041.

3. Hellström I., Raycraft J., Hayden-Ledbetter M. et al. The HE4 (WFDC2) protein is a biomarker for ovarian carcinoma. Cancer Research, 2003, vol. 63, no 13, pp. 3695-3700.

4. Moore R.G., Hill E.K., Horan T. et al. HE4 (WFDC2) gene overexpression promotes ovarian tumor growth. Scientific Reports, 2014, vol. 4, no. 1. Available at: https://www.ncbi.nlm.nih.gov/pmc/articles/PMC3880958 (accessed 14.11.2019), doi: 10.1038/srep03574.

5. Zhu Y.F., He L.S., Zhang Z.D. et al. Expression of serum human epididymal secretory protein HE4 at low grade and high grade serous carcinomas. Asian Pacific Journal of Tropical Medicine, 2012, vol. 5, no 12, pp. 925-930, doi 10.1016/s1995-7645(12)60175-8.

6. Yang X., Zhu S., Li L. et al. Identification of differentially expressed genes and signaling pathways in ovarian cancer by integrated bioinformatics analysis. OncoTargets and Therapy, 2018, vol. 11, p. 1457-1474, doi 10.2147/ott.s152238.

7. Gąsiorowska E., Walkowiak G.P., Warchoł W. et al. Ovarian cancer and normal fallopian tube high WFDC2 expression does not correlate with HE4 serum level. Polish Gynaecology, 2015, vol. 86, no 5, pp. 335-339, doi 10.17772/gp/2418.

8. Maniatis T., Frich E., Sembruk Dzh. Methods of genetic engineering. Molecular cloning, transl. from Eng., edited by Baev A.A. and Skrabin K.G [Metody geneticheskoi inzhenerii. Molekuliarnoe klonirovanie. Per. s ang. iazyka pod red. Baeva A.A. i Skriabina K.G.], Moscow, Mir, 1984, 749 p.

9. Georgakopoulos P., Mehmood S., Akalin A. et al. Immunohistochemical localization of HE4 in benign, borderline, and malignant lesions of the ovary. International Journal of Gynecological Pathology, 2012, vol. 31, pp. 517-523, doi 10.1097/pgp.0b013e31824fe269.

10. Sergeeva N.S., Alentov I.I., Marshutina N.V. Human epididymis protein He4 as a novel tumor- 
associated marker [Belok epididimisa cheloveka He4 kak novyi opukholeassotsiirovannyi marker]. Gynecologic Oncology-Onkoginekologiia, 2016, no 4, pp. 48-58.

11. Molina R., Escudero J.M., Augé J.M. et al. A novel tumors marker for ovarian cancer: comparison with CA125and ROMA algorithm in patients with gynecologicaldiseases. Tumor Biology, 2011, vol. 32, no 6, pp. 1087-1095, doi 10.1007/s13277-011-0204-3 